\title{
Charged particle velocity map image reconstruction with one-dimensional projections of spherical functions
}

\author{
Thomas Gerber, ${ }^{\text {a) }}$ Yuzhu Liu, Gregor Knopp, Patrick Hemberger, Andras Bodi, Peter Radi, \\ and Yaroslav Sych \\ Molecular Dynamics Group, Paul Scherrer Institut, 5232 Villigen, Switzerland
}

(Received 19 December 2012; accepted 11 February 2013; published online 1 March 2013)

\begin{abstract}
Velocity map imaging (VMI) is used in mass spectrometry and in angle resolved photo-electron spectroscopy to determine the lateral momentum distributions of charged particles accelerated towards a detector. VM-images are composed of projected Newton spheres with a common centre. The 2D images are usually evaluated by a decomposition into base vectors each representing the $2 \mathrm{D}$ projection of a set of particles starting from a centre with a specific velocity distribution. We propose to evaluate 1D projections of VM-images in terms of 1D projections of spherical functions, instead. The proposed evaluation algorithm shows that all distribution information can be retrieved from an adequately chosen set of 1D projections, alleviating the numerical effort for the interpretation of VMimages considerably. The obtained results produce directly the coefficients of the involved spherical functions, making the reconstruction of sliced Newton spheres obsolete. () 2013 American Institute of Physics. [http://dx.doi.org/10.1063/1.4793404]
\end{abstract}

\section{INTRODUCTION}

Electrons and ions produced in a small volume, e.g., in a photoionization process, are found on the surface of a sphere, the so-called Newton sphere, with a diameter depending on the elapsed time and the initial velocity of the charged particles. In velocity map imaging (VMI) experiments, Newton spheres are projected onto a 2D detector. ${ }^{1}$ Typically, VMI measurements yield a set of counts arranged in one or several concentric circles on a 2D pixel array (see Fig. 1). The distribution of counts belonging to a circle may exhibit an anisotropy with respect to an axis through its centre. The direction of this axis depends on the excitation process, for example on the polarization of the exciting light in gas phase photoionization. Due to the underlying angular momentum mechanics, the anisotropy is adequately described by a finite sum of Legendre polynomials defined on the polarization axis, reaching the values 1 on each circle periphery. ${ }^{2}$

The main task of evaluating VMI measurements is to restore the original Newton spheres with their specific energy and angular momentum dependency from the information provided in the recorded VM-image. Figure 1 shows two examples of VM-images registered with a delay line position sensitive device ${ }^{3}$ and a CCD camera, ${ }^{4}$ respectively. Though the eye easily discerns sets of circles with quite well defined diameters from which the energies of the constituent particles can be inferred, the numerical evaluation of the Newton sphere parameters is quite demanding. It was shown before that an inverse Able transform, the prime indicated mathematical method, generates poor numerical results as it is prone to noise, ${ }^{5,6}$ producing wrong results, especially for spheres with small diameters.

A more promising procedure is to expand the $2 \mathrm{D}$ projection which can be interpreted as a vector in $\mathbb{R}^{\mathrm{n}} \times \mathbb{R}^{\mathrm{n}}$, in terms

\footnotetext{
a)thomas.gerber@psi.ch. URL: http://rag.web.psi.ch.
}

of base vectors spanning the same vector space. Corresponding algorithms were successfully implemented and reported in recent publications. ${ }^{5,711}$ We will show that reconstruction is also possible with VM-images projected to one dimension, i.e., by an expansion with basis vectors in $\mathbb{R}^{\mathrm{n}}$.

In high count rate experiments, e.g., photoelectron photoion coincidence spectroscopy (PEPICO), ${ }^{3}$ single electrons and their associated ions have to be registered together with their time stamp. In such measurements the high required registering frequency impedes the use of $2 \mathrm{D}$ detectors with a long read-out time. For fast measurements there exist detectors basing on micro-channel plates (MCP) combined with a split anode or a delay line anode (e.g., Photonis, RoentDek Handels $\mathrm{GmbH}$ ) allowing position detection of charged particles up to a count rate of a few MHz. Both types of detectors are not well suited for recording multi-hits occurring in the dwell time of registration which amounts at least to some $50 \mathrm{~ns}$. In view of very fast detection, also coping with multi-hits, parallel registration with a multichannel pixel detector may become necessary. However, emerging 2D pixel cameras ${ }^{12,13}$ providing a time stamp for each event are still expensive or do not yet provide the spatial and temporal resolution required in high definition photoelectron spectroscopy (PES) and photoionization mass spectrometry (PIMS). To alleviate the problem of single particle momentum measurements at very high rates, as encountered, e.g., in synchrotron based experiments, we investigate the possibility to register VMI signals in real time with one or two adequately arranged fast linear detectors that may become available at much lower costs.

In fact, the currently used delay line detectors already base on 1D projections, as they intrinsically consist of a combination of two or three linear detectors, mounted at $90^{\circ}$ or $60^{\circ}$, respectively, each providing a coordinate of the hitting particle. The data obtained on each linear array correspond to a projection of the $2 \mathrm{D}$ image to the axis of the detector. Usually, the coordinates of two orthogonally mounted 
(a)

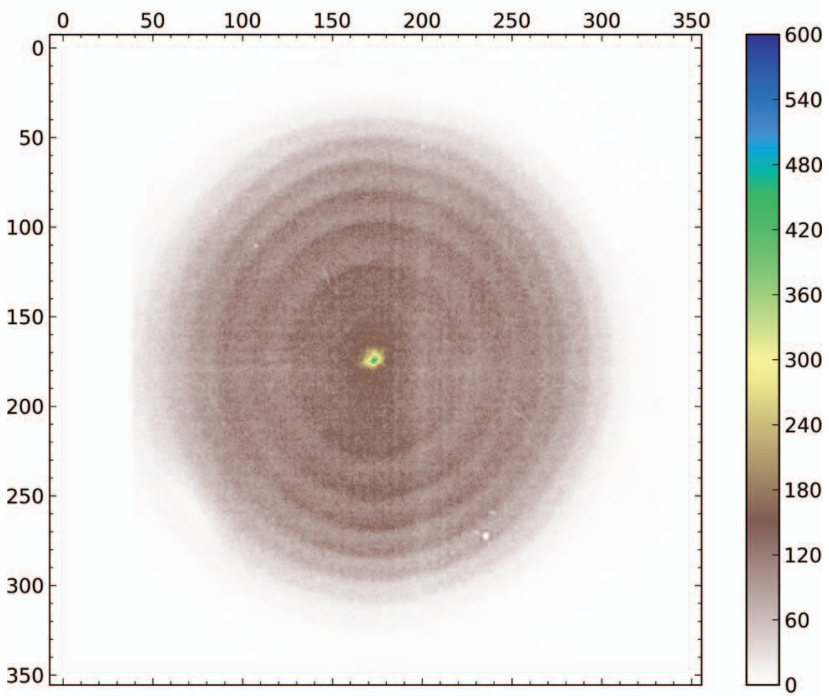

(b)

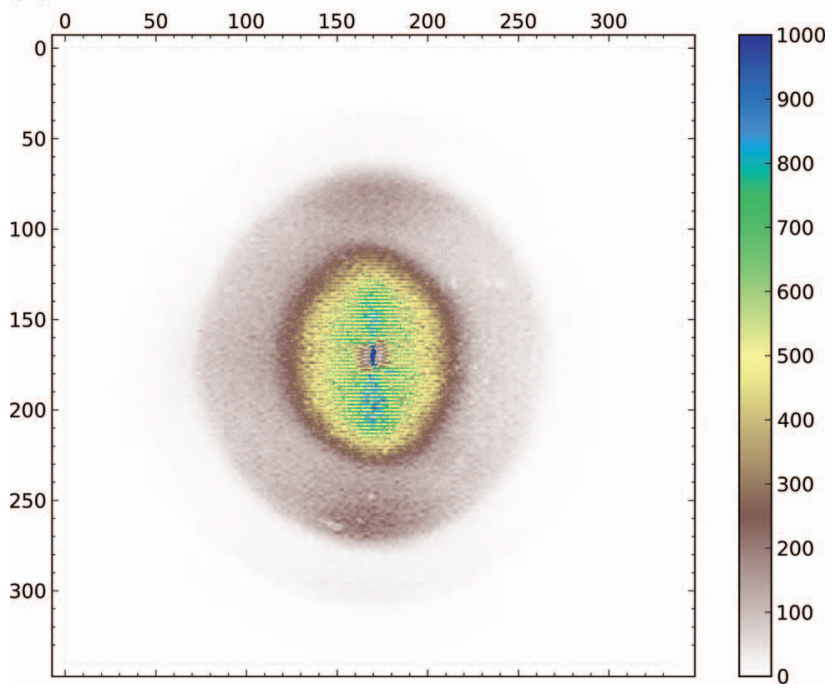

FIG. 1. Experimental VM-images. (a) Photoelectron velocity map image of $\mathrm{C}_{2} \mathrm{~F}_{4}$ recorded at SLS/VUV beamline with a photon energy of $11.55 \mathrm{eV}$. (b) Photoelectron velocity map image of $o$-xylen excited with fs pulses at center wavelengths of $400 \mathrm{~nm}$ and $800 \mathrm{~nm}$.

detectors are correlated and compiled as a 2D image - a step that becomes obsolete with the evaluation method proposed here. Instead of using an $n \times n$ pixel camera we thus propose to use the $m \times n$ pixel data obtained from $m$ independent linear $n$-pixel cameras directly as they contain the full information about the involved Newton spheres.

It will be shown below that even a single 1D projection does not necessarily imply a prohibitive loss of information. The diameters of Newton spheres can in most cases be retrieved. Even anisotropies can be evaluated within some theoretical and numerical restrictions from a single 1D projection, albeit with sometimes severe numerical restrictions. Hence, two or more linear detectors greatly improve the reconstruction in a practical experiment. The proposed evaluation scheme for 1D data, called lin-BASEX below, also opens an easy way to retrieve the momentum and angular distribu- tions from 2D VM-images, registered with a CCD camera as used in the majority of current PES/PIMS experiments.

If 2D VM-images are available, the new algorithm is not intrinsically superior to the previous evaluation methods. But, lin-BASEX has advantages in saving several evaluation steps. Lin-BASEX reduces the number of equations to be solved by a factor $n / m$ compared to a full $2 \mathrm{D}$ decomposition involving $n^{2}$ equations. A computational speed gain of a factor $(n / m)^{3}$ can result taking into account that the inversion of a matrix with range $\mathrm{N}$ alone involves $N^{3}$ multiplications. Moreover, the decomposition with Lin-BASEX produces final results without any post-processing. The common centre of the projected Newton spheres is easily and accurately determined by a convolution of the recorded projections. Lin-BASEX provides a way to check the quality of the analyzed VM-image and gives an estimate of the achievable accuracy.

\section{TYPICAL SETUP}

A VMI setup consists in essence of an electrostatic lens system that images charged particles produced in a small volume to a detector, usually an MCP-phosphor combination. Particle images on the phosphor screen can then be registered with a 2D CCD camera or another position sensitive detector. ${ }^{14,15}$

In velocity map imaging the detector is placed at the focus of a lens system. Particles with the same velocity, i.e., particles moving in the object space on parallel trajectories, are imaged by a stigmatic lens system onto one spot in the focal plane irrespective of their spatial provenance. Particles departing from the origin into the half space opposite to the one containing the detector plane will hit the same image spot as those departing directly towards the detector for a specific pair of angles. ${ }^{7}$ In a practical setup, particles starting at equal angles with respect to the flight axis into the two half spaces fulfill this condition, as long as the returning particle passes within the object circle for which the optics provide a stigmatic image.

Every realisation of an electrostatic lens system is plagued by the lack of dispersing lens elements. ${ }^{16}$ Therefore, the interaction volume in which charged particles are produced has to be kept small compared to the dimensions of the used electro-static lenses. Stigmatic imaging can only be achieved for rays with small angles in a narrow paraxial region. However, for limited interaction volumes and for small initial energies compared to those gained in the acceleration towards the detector, it is possible to set up a VMI experiment with smaller aberrations ${ }^{17-19}$ than the spatial resolution of the detector, e.g., the pixel size of a CCD camera.

In the following discussion we assume that the imaging system is "perfect" if the image of a single Newton sphere is imaged stigmatically within the spatial resolution of the used detector.

\section{MATHEMATICAL BACKGROUND}

In the absence of electric and magnetic fields, charged particles starting from a point randomly in all directions with equal initial speed are homogeneously distributed on the 


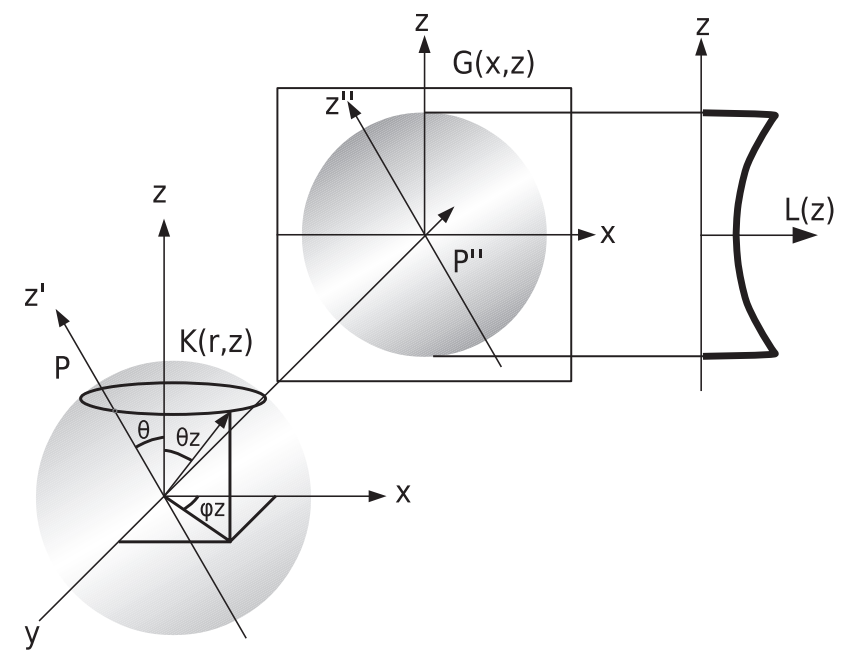

FIG. 2. Successive projections of a Newton sphere. $G(x, z)$ is the $2 D$ function registered in a typical VMI setup.

surface of a Newton sphere. The radius $r$ of the spherical surface depends on the elapsed time-of-flight $t_{\text {tof }}$ and the initial velocity, i.e., the kinetic energy of the particles, $r_{k}=\sqrt{ }\left(E_{k} / 2 m \cdot t_{\text {tof }}\right)$. A single Newton sphere $K_{k}(r)$ of particles with the same speed and starting in all directions with equal probability is isotropic and can be described by a delta function. Accordingly, a set of $n_{k}$ particles with discrete energies $E_{k}$ will be found on the peripheries of a set of concentric spheres as described in Eq. (1),

$$
K(r)=\sum_{k} n_{k} \frac{1}{4 \pi r_{k}^{2}} \delta\left(r-r_{k}\right)
$$

$K(r)$ is a probability function indicating the chance to find one particle at a specific spot on a Newton sphere. The factor $1 / 4 \pi r_{k}^{2}$ is introduced for normalisation and $n_{k}$ is the number of particles with the same kinetic energy. For continuous distributions the sum over $k$ has to be replaced by an integral. In the following discussion we assume a discrete $n_{k}$ distribution. The optics in a VMI setup map Newton spheres of the same energy but with different centres on one sphere so that Eq. (1) also applies to a real experiment with a finite interaction volume.

The electro-static lens system of a VMI setup projects Newton spheres along the coordinate $y$ (see Fig. 2) to yield the function $G(s)$ on the detector plane:

$$
\begin{aligned}
G(s) & =\sum_{k} n_{k} \frac{1}{2 \pi r_{k}} \frac{1}{\sqrt{r_{k}^{2}-s^{2}}} \Pi_{r_{k}}(s) \text { with } \\
s^{2} & =x^{2}+z^{2} \text { and } \\
\Pi_{r_{k}}(s) & =\begin{array}{ll}
1, & \text { for } 0<s<r_{k} \\
0, & \text { otherwise. }
\end{array}
\end{aligned}
$$

For an expansion of a 2D VM-image the functions defined in the right hand side of Eq. (2) are, due to their singularities, numerically ill suited as base vectors. Accordingly, current evaluation methods assume primary Newton spheres exhibiting Gaussian distributions along the radius $^{8}$ or spherical distributions according to Poisson functions. ${ }^{5}$ This assumption is mathematically acceptable as any linearly independent set of function may be used as a basis set. However, the obtained base vectors do no longer reflect the underlying physics. A physically correct approach should first determine the Newton sphere distribution of iso-energetic particles in a specific experiment. The projections of Newton spheres can be worked out by ray-tracing methods, or preferentially, by experiments with a tunable narrow bandwidth PES or PIMS signal. The obtained distribution, producing finite functions $G(s)$ would reflect the actual imaging, and thus the projection behaviour of the real apparatus. All currently used evaluation methods could resort to such real physical base vectors. However, the assumption of a straightforward basis set introduces, for typical experiments, well acceptable deviations that range below statistical variations or rounding errors introduced in the computational numerics.

No singularities appear when the Newton spheres are projected to a 1D function corresponding to a further projection of the functions $G(s)$ in Eq. (2) along the $x$-direction yielding the function $L(z)$ :

$$
L(z)=\sum_{k} \frac{n_{k}}{2 r_{k}} \Pi_{r_{k}}(|z|) .
$$

To some surprise the 1D projection of a delta Newton sphere is just a constant function over an interval of length $2 r_{k}$. A set of isotropic Newton spheres thus yields a 1D stack of stepfunctions, each with a total area of $n_{k}$, aligned around the origin. For a VM-image captured with a 2D CCD camera, Eq. (3) simply states that adding all counts in a row parallel to the $y$ axis of a projected Newton sphere yields a constant number of counts for $|z| \leq r_{k}$. The functions $\Pi_{r_{k}}$ provide a complete basis set for the expansion of the isotropic part of VM-images. In numerical evaluations we will approximate the step functions by a set of error-functions corresponding to Gaussian Newton spheres (see Appendix A). The centre of $L(z)$ corresponding to the $z$-coordinate of the centre of the associated Newton sphere can easily be determined by calculating the functions "centre of mass" or correlating the function $L(z)$ with $L(-z)$.

In PES/PIMS experiments electrons and ions will generally not depart isotropically from their origin. The distribution of charged particles on a sphere depends on the angles of the molecular dipole with respect to the direction of the exciting electric field. ${ }^{2}$ In case of randomly oriented molecular dipoles excited by light with an effective polarisation along the direction $P$, a cylindrical symmetry establishes along this axis. Thus, the anisotropy can be described by a function along a coordinate $z^{\prime}$ parallel to $P$. For a direction $P$ including an angle $\theta$ with the $z$ axis (Fig. 2), the distribution of particles on the sphere can be described by Eq. (4):

$$
K(r, z)=\sum_{k} \frac{1}{4 \pi r_{k}^{2}} \delta\left(r-r_{k}\right) \sum_{l} p_{l k} P_{l}\left(\frac{z^{\prime}}{r_{k}}\right) \Pi_{r_{k}}\left(z^{\prime}\right)
$$

with $P_{l}$ denoting Legendre polynomials of order $l$. The total number of particles having the energy $E_{k}$ evaluates to $n_{k}=$ $p_{0 k}$, as $\int P_{0}\left(z^{\prime} / r_{k}\right) \Pi_{r_{k}}\left(z^{\prime}\right) d z^{\prime}=1$ and $\int P_{l}\left(z^{\prime} / r_{k}\right) \Pi_{r_{k}}\left(z^{\prime}\right) d z^{\prime}=$ 0 for all $l \neq 0$. The distribution on each sphere with $r_{k}$ is modulated by $P_{l}\left(z^{\prime} / r_{k}\right)$ in correspondence with the dipole expansion of the ionisation process. A projection of 3D Newton spheres along the $x$ axis thus yields (see Appendix B) a quite 
compact 1D function:

$$
L(z, u)=\sum_{k} \sum_{l} P_{l}(u) P_{l}\left(\frac{z}{r_{k}}\right) \frac{\Pi_{r_{k}}(z)}{2 r_{k}} p_{l k}
$$

with $u=\cos (\theta)$. The projection angle is defined as the angle between effective polarisation and abscissa of the projection function.

Equation (5) states that a 1D projection of a VM-image comprising Legendre polynomials, contains the same Legendre polynomials with different weights depending on the projection direction. The projection-function depends only on the angle between the polarisation $\mathrm{P}$ and the projection-axis, i.e., the polarisation in Fig. 2 may also point in a direction without the $x, z$-plane (see Appendix B). Though a rotation of $\mathrm{P}$ around the $z$ axis changes the 2D projection it has no effect on the function $L(z, u)$.

All information about the involved Newton spheres and their anisotropies, i.e., all information provided by a PES/PIMS experiment are contained in Eq. (5). Looking at the functions $L(z, u)$ and $P_{l}\left(z / r_{k}\right)$ as vectors in an Euclidean vector space, Eq. (5) constitutes a system of equations expressing $L(z, u)$ as a linear combination of $P_{l}\left(z / r_{k}\right)$. As in an Euclidean vector space any vector can be approximated by a unique linear combination of linearly independent vectors (not necessarily orthogonal) there exists for a given base a unique set of coefficients $p_{l k}$, producing a least squares fit to the function $L(z, u)$.

\section{EVALUATION OF VMI DATA}

\section{A. Equation system}

In an experiment $L(z, u)$ is only known in digital form, with $n$ discrete data corresponding to the $n$ pixel in one dimension of the used detector. The function $L(z, u)$ and the polynomials $P_{l}\left(z / r_{k}\right)$ thus appear as vectors with $n$ components in $\mathbb{R}^{n}$. For Newton sphere projections according to Eq. (5), the symmetry condition $L(z, u)= \pm L(-z, u)+$ constant holds. Thus, only $n / 2$ pixels are independent. Any projection $L(z, u)$ can be decomposed in an even and an uneven part. In most PES/PIMS experiments only even Legendre polynomials $P_{l}(z)$ are expected to contribute, making $L(z, u)$ an even function around $z=0$. The experimental data can be fitted considering $L(z, u)+L(-z, u)$ as a function in the interval $0 \leq z \leq r_{k}$. If there are uneven contributions $^{8}$ the function $L(z, u)-L(-z, u)$ is different from zero and expands exclusively into uneven Legendre polynomials.

For the following discussion we assume that VM-images are registered with an $n \times n$ pixel CCD camera with columns parallel to the $z$ axis. The projection along the $x$ axis is readily available by summation of all counts in each row. We identify $z$ with its pixel number and measure the lengths $r_{k}$ in units of pixels, thus we define the discrete variables $z \rightarrow i \geq 0$, $r_{k} \rightarrow k, L(z, u) \rightarrow L_{i u}, P_{l}\left(z / r_{k}\right) \rightarrow P_{l k i}$, and $P_{l}(u) \rightarrow P_{l u}$. Equation (5) reads then:

$$
L_{i u}=\sum_{k=1}^{s} \sum_{l=0}^{q} P_{l u} P_{l k i} \frac{\Pi_{k i}}{2 k} p_{l k} .
$$

With projections in $m$ different directions $u_{m}$ we obtain $m$ systems of linear equations for the functions $L_{i u}$ with $(s \times q)$ unknown weights $p_{l k}$. Index $k$ runs to $s$, the number of Newton spheres to be resolved and $l$ runs to $q$, the number of used even or uneven Legendre polynomials. In order to avoid singularities, the summation may start with $k=1$ as all vectors $P_{l 0 i} \Pi_{1 i}$ have components $P_{l 00} \Pi_{10}=1$ and 0 elsewhere and are thus linearly dependent.

For $m$ projections with angle-cosine $u$, Eq. (6) denotes a linear equation system that can be written as $\mathbf{L}_{\mathrm{u}}=\left(P_{l u} \cdot \mathbf{B}_{1}\right)$ - $\mathbf{p}_{1}$, with $\mathbf{L}_{\mathrm{u}}$ and $\mathbf{p}_{1}$ denoting vectors containing the components $L_{i u}$ and $p_{l k}$. The $q$ matrices $\mathbf{B}_{1}=\left|\mathbf{b}_{1 \mathrm{ki}}\right|=P_{l k i} \Pi_{k i} / 2 k$ have dimension $|n / 2, s \times q|$. The $q$ triangular sub-matrices $\mathbf{B}_{l}$ contain the calculated or experimentally determined isotropic base vectors.

Numerically, a problem arises because the vectors $P_{l k i}$, with the same specific $l$, are almost collinear for two adjacent $k_{j}$, whereas the differences $D_{l k i}=P_{l(k+1) i}-P_{l k i}$ are mutually close to orthogonal. Therefore, the solution of the linear equations tend to arise as differences of adjacent contributions, i.e., in terms of $D_{l k i}$ instead of $P_{l k i}$, resulting in rapidly oscillating coefficients $p_{l k}$. Indeed, if, e.g., anisotropies are absent only the functions $\Pi_{k i}$ appear in the right hand side of Eq. (6). The difference functions $D_{l k 0}$ have an entry 1 for the pixel $i=k+1$ and zero elsewhere. The set of vectors $D_{0 k i}$ thus already constitutes a complete orthogonal basis set. A solution in terms of $n / 2$ differences $D_{0 k i}$ collapses to the trivial system $p_{l k}=k[L(k+1, u)-L(k, u)]$, i.e., $k$ times the derivative of the array $L(k, u)$. The operator $k(\mathrm{~d} / \mathrm{d} k)$ produces a mathematically correct solution, but it certainly corresponds not to a physical solution consisting of positive contributions only, there being no "negative" Newton spheres. A physical solution can often be obtained by separating Eq. (6) into single polynomial contributions, as shown below, by decreasing the resolution, i.e., averaging over 2 or more pixels or by the choice of an appropriate regularization.

\section{B. Anisotropy "peeling"}

If the number of projections is equal to the number of used Legendre polynomials, each contribution due to a specific Legendre polynomial $P_{r}$ can be singled out from Eq. (6) by choosing an appropriate linear combination of $\mathbf{L}_{\boldsymbol{u}}$ vectors. By defining the inverse matrix $P_{l u}^{-1}=\operatorname{inv}(P)_{l u}$ the following equation can be established:

$$
\sum_{u=0}^{m=q} P_{r u}^{-1} L_{i u}=\sum_{k=1}^{s} P_{r k i} \frac{\Pi_{k i}}{2 k} p_{r k}
$$

what in matrix form is $\mathbf{P}_{r u}^{-1} \cdot \mathbf{L}_{u}=\mathbf{B}_{r} \cdot \mathbf{p}_{r}$. This equation contains on the right side only the unknowns $p_{r k}$ associated to the Legendre polynomials of order $r$.

This decomposition is a potent tool for the analysis of VM-images. First it helps to find out whether there are anisotropies of any kind at all. If anisotropies are found each linear combination gives a rough estimate about the amount of the ordered contributions. In analogy to the "onion peeling" method, 7,10,20 subtracting successively Newton spheres from a VM image, the decomposition according to Eq. (7) 
can be called "anisotropy" peeling. Apart from image rotation in the case of a 2D VM-image, the evaluation of linear combinations on the left hand side of Eq. (7) does not depend on any interpretation as they result from a simple addition of raw data.

Equations (6) and (7) solve the reconstruction problem of a VM-image completely and deliver final results. With enough projections to render both equations overdetermined, the weights of all Legendre contributions are uniquely determined and constitute the parameters of each involved Newton sphere. There is no need for any post-processing such as a reconstruction of slices of the $3 \mathrm{D}$ Newton spheres. There is also no need for a preprocessing such as subtraction of dark noise from the raw data that may change the intrinsic Poisson statistics. Rather, dark images, registered immediately prior or shortly after an experiment can be evaluated with the same method. The obtained $p_{l k-\text { dark }}$ are then subtracted from the evaluated coefficients $p_{l k}$ of a VM-image.

Equation (7) is especially well suited to determine the isotropic contribution. The linear combination $\mathbf{P}_{0 u}^{-1} \cdot \mathbf{L}_{u}$ presents, apart from noise, as a stack of step-functions from which the isotropic parameters should be retrievable without ambiguity. Even if not enough projections are available to resolve each single Legendre contribution, i.e., $m<q$, it may prove beneficial to use Eq. (7) with $q^{\prime}=m<q$. Contributions with $l>q^{\prime}$ are then treated as noise. Still, the isotropic part may be retrieved with adequate accuracy as in general the higher order polynomial contribute with less weight to a real VM-image.

\section{Fewer projections than polynomials}

If there are fewer projections than Legendre contributions Eq. (6) has no unique solution for $q \times s>m \times n / 2$. Though a unique solution exists for $q \times s<m \times n / 2$, the numerically found solution is still not necessarily the physically "right" one. In order to extract the desired information the linear equation system has to be solved under restrictions that can be obtained from additional theoretical or experimental information. In this case the task to derive physically meaningful $p_{l k}$ can be tackled in two steps.

1. The number $s$ of Newton spheres is determined based on a priori knowledge and by inspection of the measured $L_{i u}$. By omission of all zero contributions from Eq. (6) a reduced set of equations remains for which $q \times s \ll m \times n / 2$. For example, with 10 Newton spheres and three involved Legendre polynomials, Eq. (6) contracts to a system of $m \times n / 2$ linear equations for 30 unknowns. The radii of the corresponding Newton spheres can in many cases be retrieved readily by solving Eq. (6) for any projection $L_{i u}$ with the isotropic basis set. It is more demanding to retrieve the number of counts in each sphere. The number of counts corresponds to the coefficient of the isotropic base vectors. All isotropic solutions have to be positive. In many cases a least square fit automatically fulfils this conditions. Otherwise, one can try a solution with half of the original base vectors, thus with reduced resolution. We also tried a set of linear programs using SAGEMATH ${ }^{21}$ and the provided cvxopt's lp-solver and glpk solver. The obtained results were unsatisfactory. Typically the linear optimization tends to neglect the contributions with the greatest radii. A more promising strategy is to isolate the isotropic contribution as well as possible. According to Eq. (7) the isotropic part can be singled out by choosing the appropriate linear combination of projections. In the case when only two projections are available one linear detector may be oriented at the magic angle of $54.7^{\circ}$ with respect to the effective polarization providing a projection with all quadratic contributions cancelled. $^{2}$

2. Determine the number $q$ of contributing Legendre polynomials according to the experimental situation. Solve with the reduced basis set containing $q \times s$ base vectors Eq. (6) or Eq. (7) to obtain all contributions. To avoid numerical instabilities we used the least square solver defined in Numpy software available within the SAGEMATH ${ }^{21}$ suite. The solver allows rejection of singular values smaller than a constant $c$ times the maximal singular value. Acceptable solutions could be obtained even in the case of $s=n / 2$ with $c=0.005$. In calculations retaining only base vectors corresponding to contributing spheres, often no conditioning was necessary. Instead of solving the equation for all projections with $q \times s$ base vectors simultaneously a better numerical stability may also be achieved by solving Eq. (6) iteratively with basis sets $\mathbf{B}_{0}, \mathbf{B}_{2}, \ldots$ subtracting $\left(B_{l u} \cdot \mathbf{B}_{l}\right) \cdot \mathbf{p}_{l}$ from $\mathbf{L}_{u}$ in each step.

Most current experiments, including those working with delay line position sensitive devices, provide at least two projections. In those cases Eq. (6) should first be solved for 2 polynomial contributions, e.g., $l=0,2$. If a positive result is obtained the contributions with $l=0,2$ can be subtracted from the projections before entering them into an equation system for $l>2$.

\section{Performance: Artificial VM-image}

The following evaluations with lin-BASEX were all performed with SAGEMATH. ${ }^{21}$ A worksheet for centring rawimages, calculation of projections at an arbitrary angle from a 2D-image, checking symmetry and solving Eqs. (6) and (7) is available as supplementary material. ${ }^{22}$

Figure 3 shows an artificial VM-image containing the 2D projection of eight Newton spheres with different radii. The trace on the right depicts a $1 \mathrm{D}$ projection orthogonal to the polarization direction, i.e., $\theta=0$. The number of counts $\beta_{0 k}$ $=n_{k}$ in each sphere varies between 160000 and 320000 . The particles were distributed on each sphere with a Gaussian distribution centred at $r_{k}$ having a width of about 2 pixel $(\sigma=1$ pix). Table I summarizes the input parameters for each sphere. The coefficients $\beta_{2}$ and $\beta_{4}$ indicate the anisotropy associated to each sphere. The spheres no. $4-6$ with radii differing only by four or five pixels appear hardly resolved in Fig. 3.

Table II shows solutions according to Eq. (6) for an increasing number of projections. The number of polynomials to expand with was set to 2 and 3 in the case of one projection and to 3 for two and three projections. The results for three projections were calculated at full resolution without any regularization according to Eq. (7). All other cases 


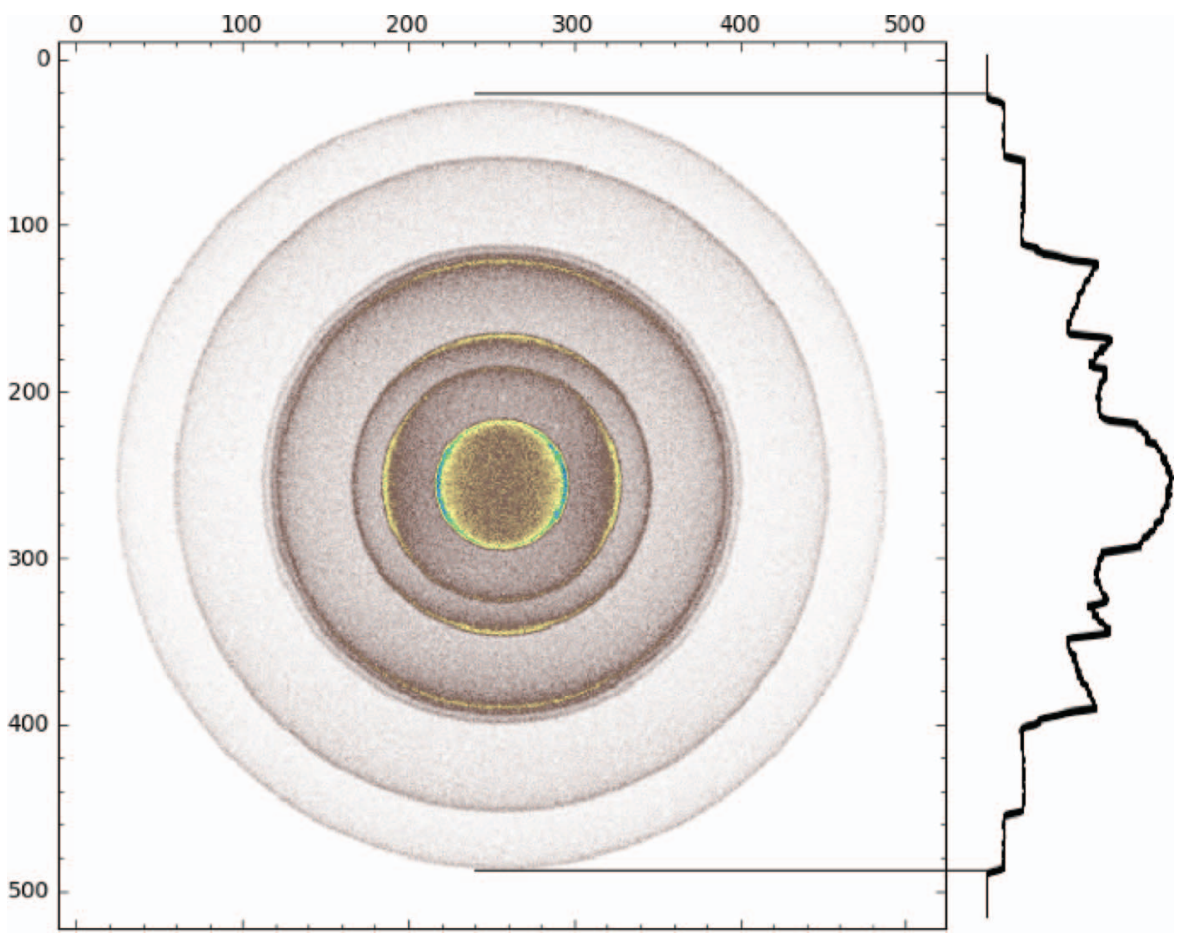

FIG. 3. Simulated VM-image with a projection at an angle of $0^{\circ}$. The VM-image comprises the contribution from 8 spheres of different anisotropy.

were evaluated with Eq. (6) and a regularization of 0.005 . The table shows the quotients of the input and output values for all spheres in the case of three projections. Normalization to one was obtained by division with the average quotient. For evaluations using one or two projections only the standard deviations of quotients are listed. Best performance is clearly obtained for 3 polynomials using three projections.

The accuracy of decomposition into base vectors hinges on the number of counts that are attributable to each sphere. An isotropic sphere with $n_{1}$ counts appears in its projection as a step-function with a step of $n_{1} / k_{1}$, with $k_{1}$ its radius. A second sphere with $n_{2}$ counts and radius $k_{2}<k_{1}$ piles upon the first step-function with height $n_{2} / k_{2}$. Any error in the determination of $n_{1} / k_{1}$ will be balanced by adjusting $n_{2} / k_{2}$ in a least square fit. The step $n_{1} / k_{1}$ can only be determined with an accuracy corresponding to the number $\Delta n_{1}=n_{1}\left(k_{1}-k_{2}\right) / k_{1}$. The relative error in determining the number $n_{1}$ is thus $n_{1}^{1 / 2} / \Delta n_{1}$, - an error that, for closely adjacent spheres, i.e., $k_{1}-k_{2} \ll k_{1}$, is much higher than the expected $n_{1}^{1 / 2} / n_{1}$ derived from Poisson statistics. For spheres no. 4 to no. 6 in Fig. 3 the expected rel-

TABLE I. Input parameters for simulated VM-image.

\begin{tabular}{lclll}
\hline \hline Sphere No. & Radius (pixel) & $\beta_{0}$ & $\beta_{2}$ & $\beta_{4}$ \\
\hline 1 & 38 & 1.2 & -0.4 & 0 \\
2 & 70 & 1.5 & -1 & 0.5 \\
3 & 90 & 1.5 & 1 & 0.4 \\
4 & 134 & 2 & 1 & 0.4 \\
5 & 138 & 1.8 & 0.5 & 0 \\
6 & 143 & 1 & 0.5 & 0.25 \\
7 & 196 & 2 & 1 & -0.5 \\
8 & 230 & 2 & 1 & -0.5 \\
\hline \hline
\end{tabular}

ative error is thus in the order of $10 \%$, in agreement with the observed deviations of the quotients $Q_{0}$ listed in Table II.

\section{E. Performance: Experimental VM-image}

Lin-BASEX was used to evaluate a PES measured with the iPEPICO setup installed at SLS/VUV beamline. ${ }^{3}$ Figure 1(a) shows an electron spectrum of $\mathrm{C}_{2} \mathrm{~F}_{4}$ at a photon energy of $11.55 \mathrm{eV}$. The image is composed of about $2.5 \times 10^{6}$ counts registered with a RoentDek DLD40 delay line detector installed at the image plane of an electron time of flight (TOF) apparatus. The VM-image with a resolution

TABLE II. Evaluated coefficients using 1, 2, and 3 projections. The quotient $Q_{i}=\beta_{i, i n} / \beta_{i, \text { calc }}$ between input and calculated coefficients is given for each sphere in the case of 3 projections. For a perfect reproduction the quotient is one. For other projections only the standard deviation over all quotients of one kind is given.

\begin{tabular}{lcccl}
\hline \hline Projections & & $Q_{0}$ & $Q_{2}$ & $Q_{4}$ \\
\hline $1\left(0^{\circ}\right)$ & Std. dev. & 0.27 & 0.69 & \\
$1\left(0^{\circ}\right)$ & & 0.23 & 2.81 & 1.37 \\
$1\left(54^{\circ}\right)$ & & 0.13 & $\ldots$ & 0.69 \\
$2\left(0^{\circ}, 90^{\circ}\right)$ & & 0.21 & 0.82 & 0.94 \\
$3\left(0^{\circ}, 90^{\circ}, 54^{\circ}\right)$ & & 0.06 & 0.13 & 0.09 \\
$3\left(0^{\circ}, 90^{\circ}, 54^{\circ}\right)$ & In/out, s $=1$ & 0.97 & 0.96 & 0 \\
& 2 & 0.98 & 0.98 & 0.98 \\
& 3 & 1.01 & 1.03 & 1 \\
& 4 & 1.07 & 0.99 & 0.97 \\
& 5 & 0.89 & 0.78 & 0 \\
& 6 & 1.08 & 1.26 & 1.21 \\
& 7 & 1 & 1.01 & 1.04 \\
& 8 & 1 & 1.02 & 1.03 \\
\hline \hline
\end{tabular}




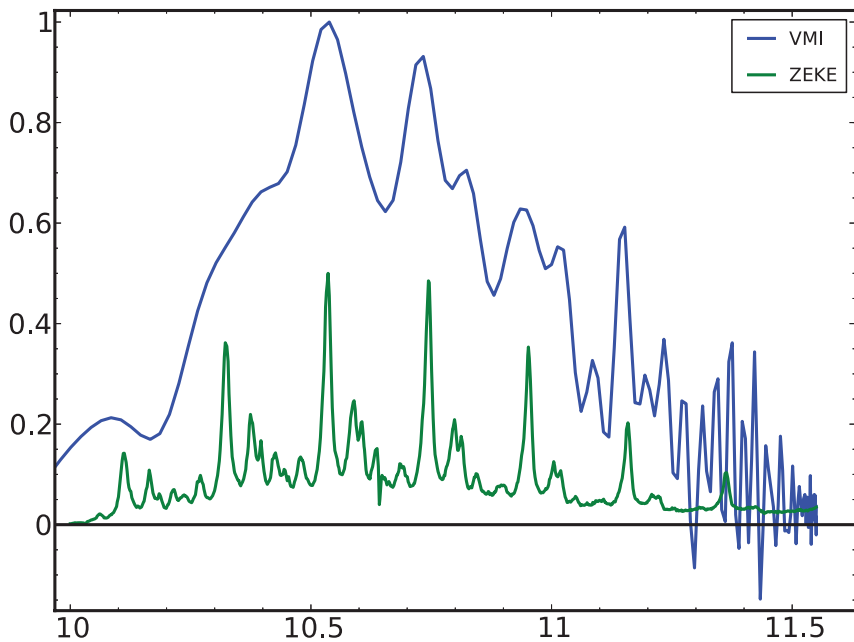

FIG. 4. Photo-electron spectrum of $\mathrm{C}_{2} \mathrm{~F}_{4}$ derived from the VM-image of Fig. 1(a). A ZEKE spectrum (lower trace) registered at SLS/VUV beamline with a resolution of $\sim 2 \mathrm{meV}$ is shown for comparison. The coordinate indicates normalized numbers of electrons versus (photon-energy - electronenergy) (PES), and photon-energy (ZEKE), respectively.

of $348 \times 348$ pixel covers a spectral range of $2.2 \mathrm{eV}$. The TOF setup was primly optimized for the detection of zero electron kinetic energy (ZEKE) electrons arriving at the centre of the detector. For low extraction fields around $100 \mathrm{Vcm}^{-1}$, a ZEKE resolution of better than $1 \mathrm{meV}$ can be realized. Electro-static optics simulations indicate a resolution of $\sim 2.5 \% \Delta E / E$ over the entire image plane. In the used configuration, at an extraction field of $180 \mathrm{Vcm}^{-1}$, the resolution thus drops from the centre to about $50 \mathrm{meV}$ at the outer rim.

Anisotropy-peeling, including even and uneven Legendre polynomials, unveil that the obtained anisotropic coefficients for Fig. 1(a) contain no spectral information. This is how it should be, because an electron leaves a $\pi$ orbital as an s wave. Thus, the image is evaluated in terms of isotropic contributions only. Electron speeds were derived from a projection along the vertical axis using Eq. (6). The photoelectron spectrum versus electron energy, i.e., the square of the electron speeds scaled to the corresponding energies, is depicted in Fig. 4. The lower trace represents a ZEKE spectrum taken with the same setup at a resolution of $2 \mathrm{meV}$. The assignment of the spectrum to vibrational bands of $\mathrm{C}_{2} \mathrm{~F}_{4}$ can be found in Ref. 23 Very recently, the assignment was revisited by Harvey et al. ${ }^{24,25}$

Comparison of the two spectra in Fig. 4 indicate a promising performance of the electron VM-setup as an electron spectrometer, beyond its design as ZEKE detector. The VM image exhibits a moire stemming from a, most likely electronic, instrumental deficiency. Projections at different angles indicate an elliptical distortion due to a different scale along the vertical and horizontal axis. An improved imaging performance of the electron TOF component was realised in the new $\mathrm{i}^{2} \mathrm{PEPICO}^{26}$ setup to be used as an electron spectrometer. The data of the delay line detector, providing 1D Newton sphere projections, can then be used directly without prior compilation of a 2D VM-image.

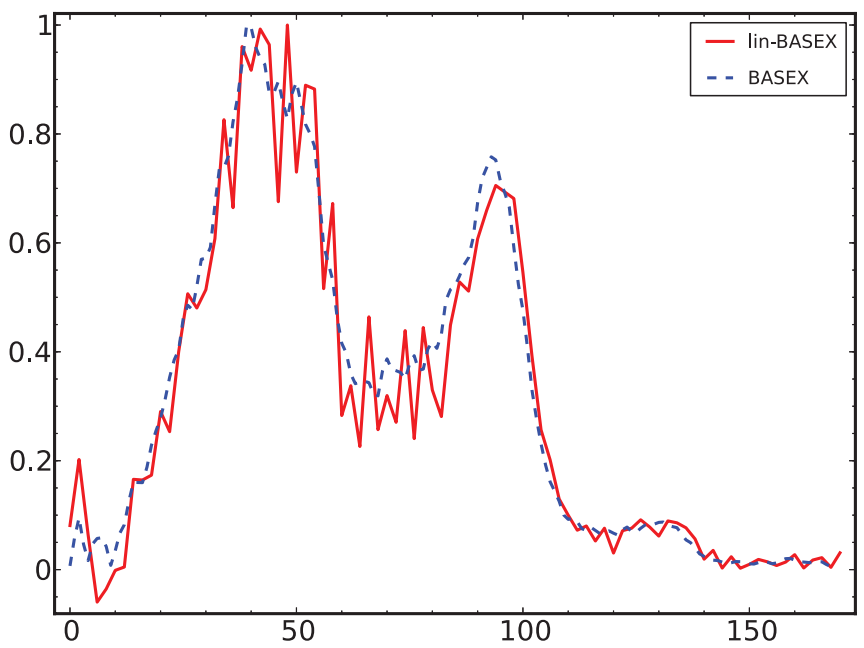

FIG. 5. Electron speed distribution in o-xylen excited with fs-pulses at 400 $\mathrm{nm}$ and $800 \mathrm{~nm}$. Normalized number of particles (coordinate) versus velocity (abscissa) in pixel coordinates. 124 pixel correspond to an electron speed of $10^{6} \mathrm{~m} / \mathrm{s}$, resp., to $2.85 \mathrm{eV}$ electron energy. The solid trace obtained with linBasex exhibits negative contributions for small speeds. The dotted (blue) line indicates the speeds evaluated with BASEX.

As a second example the VM-image depicted in Fig. 1(b) is analysed with BASEX ${ }^{5}$ and lin-BASEX. It was produced by femtosecond time-resolved photoelectron imaging in a study on the dynamics of excited $o$-xylen. The VM-image was captured by a CCD camera (MS-2821E, Mintron) providing a resolution of $n=340$ pixels. In this work ${ }^{4}$ anisotropies were evaluated up to the sixth order. The results found in second order, (i.e., the coefficients $\beta_{2}$ ) were used to assign the time dependent spectrum to specific transitions in $o$-xylene. The shown VM-image is now explored up to the highest physically indicated 6 th order ( 3 photons, $J_{\max }=3$ ) using BASEX and lin-BASEX.

Figure 5 shows the distribution of electrons as a function of their velocity evaluated with both methods. Lin-BASEX, produces negative contributions for spheres with small radii. These contributions could not be avoided completely by choosing a lower resolution ( 85 base vectors, as shown in Fig. 5), by rejection of singular values nor by clipping off base vectors associated to small radii. Anisotropy peeling according to Eq. (7) was employed to separate the 4 polynomial contributions using 4 projections at $5^{\circ}, 90^{\circ}, 54.7^{\circ}$, and $30^{\circ}$. The projections were calculated by adding rows after rotating the image by the desired angle using the Scipy "ndimage" package running under SAGEMATH. ${ }^{21}$ The linearly combined projections are shown in Fig. 6. The uppermost trace indicates the total number of counts as a function of the distance from the centre. The other traces indicate the number of counts that have to be added or subtracted from the total to account for the anisotropic contribution of the specific order.

The separated equation systems were solved with 170 (full resolution) and 85 base vectors, respectively. In the case of $n / 2=170$ vectors, regularization was necessary. Singular values smaller than 0.005 times the maximal value had to be rejected. The results depend slightly on the choice of singular value cutoff. Solving the equation systems with 85 base vectors produced stable solutions without any regularization. 


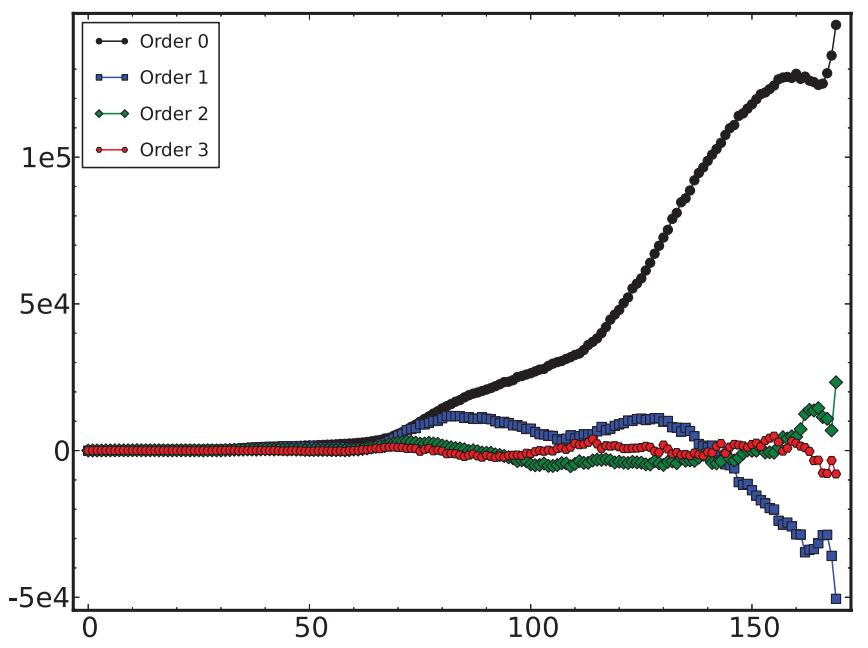

FIG. 6. The traces are linear combinations of projections of the raw VMimage showing number of counts versus distance (in pixel) from the centre at right. Only one half of each projection is shown. The centre of the Newton spheres at pixel 171 with a very high value is omitted from the plots. Each trace contains anisotropic contributions of only one kind. The curve (black) with positive values only indicates the total number of counts (order zero). The curve with the greatest positive and negative excursions (blue) corresponds to second order, the trace with smaller values (green) to the sixth order. The paraxial trace (red) indicates forth order contributions.

The solutions corresponding to spheres with small radii (up to $r=16$ pixels) are not interpretable. Actually, as obvious from Fig. 1(b), the central region shows big anisotropic variations that do not find a physical explanation. Moreover, the picture exhibits a horizontal moire. The moire is averaged out using a projection at $5^{\circ}$ instead of $0^{\circ}$.

In a further step, $\beta$-values of each kind were summed up for spheres with radii $20-48,49-65$, and 70-110 pixels corresponding to transition bands, numbered $1,2,3$, in $o$ xylen. The obtained results are listed in Table III. For comparison, the reconstructed sliced spheres using BASEX were cast as functions of their polar angle. Functions within the same band of spheres, were again summed up to produce three polar functions for the same transitions in $o$-xylen (for details see Ref. 4). The anisotropy coefficients $\beta$ obtained from these three functions are also added to Table III.

The obtained solutions unveil that the contributions decrease with higher order as expected from the underlying angular momentum mechanics. The difference of $\beta$-values in

TABLE III. Anisotropy coefficients obtained with BASEX and lin-BASEX.

\begin{tabular}{lcccc}
\hline \hline & $\begin{array}{c}\text { Band } \\
\text { (pixel range) }\end{array}$ & $\beta_{2}$ & $\beta_{4}$ & $\beta_{6}$ \\
\hline BASEX & $1,(20-48)$ & 0.43 & 0.22 & 0.30 \\
& $2,(49-66)$ & 0.72 & 0.21 & 0.13 \\
& $3,(67-110)$ & 0.96 & 0.85 & 0.48 \\
lin-BASEX & 1 & 0.43 & 0.39 & 0.33 \\
4 projections & 2 & 0.81 & 0.44 & 0.24 \\
& 3 & 0.87 & 0.67 & 0.22 \\
lin-BASEX & 1 & 0.42 & 0.28 & 0.28 \\
179 projections & 2 & 0.80 & 0.32 & 0.18 \\
& 3 & 0.87 & 0.83 & 0.32 \\
\hline \hline
\end{tabular}

both sets amounts up to 0.2 what can be considered as a fair agreement enabling a qualitative interpretation of the experiment, e.g., the assignment of transitions according to their character. However, the observed discrepancies impede the quantitative use of the obtained $\beta$-coefficients in either case. In contrast to BASEX, lin-BASEX shows immediately that the investigated VM-image departs from symmetry. According to Eq. (6), projections at angles $\pm \theta, 180^{\circ} \pm \theta$, should coincide. Hence, a comparison of any four equivalent projections can be used to check symmetry. The four projections of Fig. 1(b) with $\theta=30^{\circ}$ differ locally up to $25 \%$.

Both evaluation methods are quite sensitive on the choice of the centre. BASEX does not provide an algorithm to determine the centre of a VM-image. The centre can be found heuristically by optimizing resolution. Finding the centre of a VM-image is straightforward with lin-BASEX as described above. The centres derived from convolutions of 1D projections differed from the centre of gravity of Fig. 1(b) by up to 5 pixels. Higher order $\beta$-coefficients change up to \pm 0.2 upon shifting the centre by a few pixels. The deviations of corresponding values in Table III thus reflect rather the two methods different treatment of systematic errors than a different physical interpretation. Lin-BASEX produces an average over the whole VM-image equivalent to BASEX if $\sim n / 2$ projections are used. Indeed, with 179 projections (see Table III) the numbers obtained with BASEX are closely matched. However, BASEX averages over all asymmetries due to instrumental deficiencies without warning, whereas lin-BASEX provides a guess about the quality of the explored VM-image. For a safe quantitative interpretation, the explored VM-image shown in Fig. 1(b) does not have the required quality. We tend to prefer the lin-BASEX method for another advantage: Regularization schemes can in many cases be avoided. Hence, there is no averaging involved besides choosing a lower than possible resolution. BASEX invokes Tikhonov regularization by default, introducing a hardly interpretable kind of averaging, unless carefully accounted for. ${ }^{9}$ Actually, we also observed a dependency of the lin-BASEX results on the rejection of singular values in the calculations with full resolution. Thus avoiding any regularization is certainly of merit.

\section{DISCUSSION}

The projection of Newton spheres to a plane yields a function $G(x, z)$ defined over a circular region. It can be derived from the underlying physics that this function is a superposition of a finite number $q \times s$ functions, with $q$ denoting the number of involved Legendre polynomials and s the number of spheres. Decomposition of a VM-image into base vectors derived from 1D projections of spherical functions requires that the isotropic contributions are positive or zero. A straightforward decomposition with a least-square fit may result in negative contributions that can not be interpreted physically. Non-physical solutions typically result when evaluating VM-images containing a rather low number of counts.

Apart from numerical instabilities, indicating the use of SVD solvers ${ }^{8}$ or Tikhonov regularization, ${ }^{5}$ the conventional evaluation procedures using $2 \mathrm{D}$ base functions are expected 
to be more robust than lin-BASEX. The number of the $n / 2$ $\times n / 2(n=$ number of pixel) data from a 2D VM-image is much higher than the number of typically used base vectors. In BASEX, ${ }^{5}$ the number of base vectors is reduced to $n$ by choosing base vectors as a direct product of two basis sets, one for radii $(n / 2)$ and another for the $z$-dependence $(n / 2)$. The number of equations, for each pixel one equation, is retained. With such a high redundancy, $n^{2}$ equations for $n$ unknowns, the regularized numerical least-square problem is likely to find a stable solution. In contrast to this expectation we observed that also lin-BASEX, stating much less equations, yields readily meaningful solutions-in many cases even without any regularization.

Roberts et al. ${ }^{10}$ decompose VM images into $n / 2$ functions that can be evaluated in terms of $q$ Legendre polynomials. Intrinsically they thus use $n / 2 \times q$ base vectors with as many unknowns. Among all models up to now, polar onion peeling makes the intuitive best use of the available data in terms of the underlying physical interpretation. The problem of "positive contributions" is solved by omitting negative values in the proposed differencing procedure. This somewhat arbitrary omission, constituting a data loss, does apparently not lead to instabilities, unless very sparse VM images are evaluated.

With $m$ projections, lin-BASEX reduces the $n \times n$ equations of BASEX to $n / 2 \times m$ equations, with $m$ typically smaller than 4 , thus decreasing redundancy considerably. If $n$ projections were used lin-BASEX becomes mathematically equivalent to BASEX. Under this aspect, the main message here is, that not $n$ projections, but only as many as the $q$ polynomial contributions, are needed to unambiguously decompose a VM-image up to the order $2 q$ for even and $2 q+1$ for uneven projections. If the basis set can further be reduced, e.g., by inferring the radii of contributing spheres from experiment or by a preliminary lin-BASEX evaluation, even less than $q$ projections may yield a useful result.

The measured 1D projections in lin-BASEX serve directly to determine the centre of the constituent Newton Spheres. As 1D projections can also be obtained easily from a 2D VM-registering, convolution of 1D projections is recommended for centring VM-images in all cases. Alternatively for high quality VM-images the centre can readily be retrieved by determination of the centre of gravity.

Linear combinations of projections at different angles provide a means to separate anisotropies by their order and thus provide a direct visual control of the character of single Newton spheres. In contrast to onion peeling, anisotropypeeling does not change the remaining data set in every peeling step. Lin-BASEX decomposes the data set as a whole into its components without any lossy data manipulations. The decomposition is therefore independent on the chosen resolution, while in onion peeling progressing decompositions depend on the averaging of each precedent digitized rim. Though also BASEX preserves the integrity of raw data entered into a solver, lin-BASEX produces final results whereas BASEX solutions have to be further developed into slices of the 3D Newton spheres. The solutions of lin-BASEX are physical quantities, numbers of particles and coefficients of Legendre contributions, that can immediately be attributed to each single Newton sphere involved.

\section{ACKNOWLEDGMENTS}

This work was supported by the Swiss National Centre of Competence in Research, Molecular Ultrafast Science and Technology (NCCR-MUST) and the Swiss Federal Office of Energy (BFE).

\section{APPENDIX A: PROJECTION OF NEWTON SPHERES TO ERROR FUNCTIONS}

If the primary Newton sphere is assumed as resulting from a Gaussian distribution of energies (cf., Ref. 8) around $r_{k}$ an approximate basis system similar to Eq. (3) can be found. Adding Newton spheres with radii $r^{\prime}{ }_{k}$ according to a Gaussian distribution around $r_{k}$ within a width $\delta$ results in

$$
\begin{aligned}
L(z) & =\sum_{k} \int_{-\infty}^{z} n \frac{\left.\mathrm{e}^{r_{k}^{\prime}-r_{k}} \delta\right)^{2}}{2 r_{k}^{\prime}} \Pi_{r_{k}^{\prime}}(|z|) \delta r_{k}^{\prime} \\
& \approx \sum_{k} \frac{n}{2 r_{k}} \Pi_{r_{k}}(|z|) \int_{-\infty}^{z} \mathrm{e}^{\left(\frac{r_{k}^{\prime}-r_{k}}{\delta}\right)^{2}} \delta r_{k}^{\prime} \\
& =\sqrt{\pi} \sum_{k} \frac{n}{2 r_{k}} \operatorname{erf}\left(\left|\frac{z-r_{k}}{\delta}\right|\right)
\end{aligned}
$$

for $\delta \ll r_{k}$. Thus, error functions are appropriate base vectors. For numerical simulations the erf function in Scipy ${ }^{19}$ was used to construct the isotropic base vectors. Using erf with smooth steps leads to numerical instabilities as the scalar product between adjacent base vectors is greater than the one between step functions, i.e., the vectors become more collinear. Optimal performance was obtained with a sharp step $(\delta=0.001)$.

\section{APPENDIX B: PROOF OF EQ. (5)}

For the following discussion we assume that a VM image is registered with a detector parallel to the $z$ axis or with a $2 \mathrm{D}$ detector in the $x z$-plane. The question arises what is the projection if the polarisation vector has an arbitrary direction in respect to the detector frame?

Let first the polarisation lie in the $x z$-plane and point along an axis $z^{\prime \prime}$ in a coordinate system $z^{\prime \prime}, x^{\prime \prime}$ with the angle $\theta=\varepsilon$ between $z^{\prime \prime}$ and $\mathrm{z}$, (see Fig. 2).

$$
\begin{aligned}
x^{\prime \prime} & =e \cdot x-f \cdot z, \\
z^{\prime \prime} & =f \cdot x+e \cdot z, \\
e & =\cos (\epsilon), \quad f=\sin (\epsilon) .
\end{aligned}
$$

This corresponds to a polarization vector turned counterclockwise by the angle $\varepsilon$ from the $z$ axis. With a distribution as given in Eq. (4), the anisotropic analogue of Eq. (2) reads

$$
\begin{aligned}
G(s) & =\sum_{k} \frac{p_{l k}}{2 \pi r_{k}} \frac{P_{l}\left(\frac{z^{\prime \prime}}{r_{k}}\right)}{\sqrt{r_{k}^{2}-s^{2}}} \Pi_{r_{k}}(s) \text { with } \\
s^{2} & =x^{2}+z^{2} \text { and } \\
\Pi_{k}(s) & =\begin{array}{ll}
1, & \text { for } 0<s<k \\
0, & \text { otherwise. }
\end{array}
\end{aligned}
$$


Integration along axis $x$ yields for every summand in Eq. (B2),

$$
\begin{aligned}
& \text { With } a=\sqrt{r_{k}^{2}-x^{2}}, \\
& \frac{p_{l k}}{2 \pi r_{k}} \int_{-\infty}^{\infty} \frac{P_{l}\left(\frac{z^{\prime \prime}}{r_{k}}\right)}{\sqrt{r_{k}^{2}-s^{2}}} \Pi_{k}(s) d x \\
& =\frac{p_{l k}}{2 \pi r_{k}} \int_{-a}^{a} \frac{P_{l}\left(\frac{z^{\prime \prime}}{r_{k}}\right)}{\sqrt{r_{k}^{2}-x^{2}-z^{2}}} d x \\
& =\frac{p_{l k}}{2 r_{k}} P_{l}(e) P_{l}\left(\frac{z}{k}\right)
\end{aligned}
$$

from which Eq. (5) can be inferred for the case that the polarization lies within the $x z$-plane. Equation (B3) can be proven for $l=0,1,2, \ldots$ by direct integration using for instance a computer algebra system (CAS, Maxima). ${ }^{19}$ We could not find an analytical proof of Eq. (B3) for arbitrary indices $l$.

However the same result, even for an arbitrary orientation of the polarisation vector, follows from a more general approach. Each component of the distribution defined in Eq. (4) can be expressed in terms of spherical functions. Using the notation of Zare, ${ }^{2}$ a spherical function $Y_{L 0}\left(\theta_{z^{\prime}}, \varphi_{z^{\prime}}\right)$ transforms under a rotation $R(\varphi, \theta, \chi)$ as

$$
R \cdot Y_{L M}\left(\theta_{z^{\prime}}, \phi_{z^{\prime}}\right)=\sum_{M^{\prime}} D_{M^{\prime} M}^{L}(\phi, \theta, \chi) \cdot Y_{L M^{\prime}}\left(\theta_{z}, \phi_{z}\right),
$$

where $\left(\theta_{z^{\prime}}, \varphi_{z^{\prime}}\right)$ refers to the angles in the "experimental" $x^{\prime} y^{\prime} z^{\prime}$ coordinate-systems with a polarization in $z^{\prime}$ direction and $\left(\theta_{z}, \varphi_{z}\right)$ to the "detector" frame $x y z$ obtained by rotation $R$. $D_{M^{\prime} M}^{L}$ denotes the rotation matrix in Euler angles. Every distribution component of an anisotropic Newton sphere with polarization direction $\mathrm{z}^{\prime}$ corresponds to a real spherical function $Y_{L 0}\left(\theta_{z^{\prime}}, \varphi_{z^{\prime}}\right)$, i.e., $M=0$. This "experimental" distribution appears in the direction $z$ as a sum of spherical functions $Y_{L M^{\prime}}\left(\theta_{z}, \varphi_{z}\right)$ with the same $L$ and $-L<M^{\prime}<L$.

The projection of $Y_{L 0}\left(\theta_{z^{\prime}}, \varphi_{z^{\prime}}\right)$ to a $1 \mathrm{D}$ function $L(z)$ corresponds to the integral along the angle $\varphi$ from 0 to $2 \pi$ in the $x y z$ frame. In order to perform the integration of $Y_{L M}\left(\theta_{z^{\prime}}, \varphi_{z^{\prime}}\right)$ along the angle $\varphi_{z}$ we use the transform given in Eq. (B4) to obtain:

$$
\begin{aligned}
& \int_{0}^{2 \pi} Y_{L O}\left(\theta_{z^{\prime}}, \phi_{z^{\prime}}\right) d \phi_{z} \\
& \quad=\sum_{M^{\prime}} \int_{0}^{2 \pi} D_{M^{\prime} 0}^{L}(\phi, \theta, \chi) Y_{L M^{\prime}}\left(\theta_{z}, \phi_{z}\right) d \phi_{z} .
\end{aligned}
$$

From a more explicit form of Eq. (B5), using the definitions for the spherical functions $Y$ and the rotation matrix $D$ in Ref. 2, it follows readily that only the term with $M^{\prime}=0$ survives integration.

$$
\begin{aligned}
& \int_{0}^{2 \pi} Y_{L O}\left(\theta_{z^{\prime}}, \phi_{z^{\prime}}\right) d \phi_{z} \\
& \quad=\sum_{M^{\prime}} \int_{0}^{2 \pi} e^{-i \phi M^{\prime}} d_{M^{\prime} 0}^{L}(\theta) e^{-i(0) \chi} e^{i \phi_{z} M^{\prime}} \Theta_{L M^{\prime}}\left(\theta_{z}\right) d \phi_{z} \\
& \quad=2 \pi d_{0,0}^{L}(\theta) \Theta_{L O}\left(\theta_{z}\right)
\end{aligned}
$$

By substitution of the matrix elements $d$ and the functions $\Theta$ (tabulated in Ref. 2)

$$
\begin{gathered}
d_{0,0}^{L}(\theta)=P_{L}(\cos \theta), \\
\Theta_{L O}\left(\theta_{z}\right)=c_{L} P_{L}\left(\cos \theta_{z}\right)
\end{gathered}
$$

and inserting a constant $c_{\mathrm{L}}$ to adapt to the norm used here, Eq. (5) can be inferred to be valid for polarisations in arbitrary direction. There is no $\varphi$ dependency, and any 1D-projection depends only on the angle $\theta$ between the polarisation vector and the axis onto which the Newton sphere is projected. However, the range in which $\theta$ varies upon rotation of the projection axis within the $x z$-plane does depend on the angle $\varphi$. If $\varphi=\theta=90^{\circ}$, i.e., the polarization vector is orthogonal to the detector plane, there is no change of $\theta$ for any direction in the $x z$-plane and all projections become equivalent.

${ }^{1}$ A. T. J. B. Eppink and D. H. Parker, Rev. Sci. Instrum. 68, 3477 (1997).

${ }^{2}$ R. N. Zare, Angular Momentum: Understanding Spatial Aspects in Chemistry and Physics (Wiley, 1988).

${ }^{3}$ A. Bodi, M. Johnson, T. Gerber, Z. Gengeliczki, B. Sztáray, and T. Baer, Rev. Sci. Instrum. 80, 034101 (2009).

${ }^{4}$ Y. Liu, B. Tang, H. Shen, S. Zhang, and B. Zhang, Opt. Express 18, 5791 (2010).

${ }^{5}$ V. Dribinski, A. Ossadtchi, V. A. Mandelshtam, and H. Reisler, Rev. Sci. Instrum. 73, 2634 (2002).

${ }^{6}$ S. Ma, H. Gao, L. Wu, and G. Zhang, J. Quant. Spectrosc. Radiat. Transf. 109, 1745 (2008).

${ }^{7}$ J. Winterhalter, D. Maier, J. Honerkamp, V. Schyja, and H. Helm, J. Chem. Phys. 110, 11187 (1999).

${ }^{8}$ G. A. Garcia, L. Nahon, and I. Powis, Rev. Sci. Instrum. 75, 4989 (2004).

${ }^{9}$ F. Renth, J. Riedel, and F. Temps, Rev. Sci. Instrum. 77, 033103 (2006).

${ }^{10}$ G. M. Roberts, J. L. Nixon, J. Lecointre, E. Wrede, and J. R. R. Verlet, Rev. Sci. Instrum. 80, 053104 (2009).

${ }^{11}$ K. Zhao, T. Colvin, W. T. Hill, and G. Zhang, Rev. Sci. Instrum. 73, 3044 (2002).

${ }^{12}$ C. Cammi, F. Panzeri, A. Gulinatti, I. Rech, and M. Ghioni, Rev. Sci. Instrum. 83, 033104 (2012).

${ }^{13}$ P. Trueb, B. A. Sobott, R. Schnyder, T. Loeliger, M. Schneebeli, M. Kobas, R. P. Rassool, D. J. Peake, and C. Broennimann, J. Synchrotron Radiat. 19, 347 (2012).

${ }^{14}$ M. N. R. Ashfold, N. H. Nahler, A. J. Orr-Ewing, O. P. J. Vieuxmaire, R. L. Toomes, T. N. Kitsopoulos, I. A. Garcia, D. A. Chestakov, S.-M. Wu, and D. H. Parker, Phys. Chem. Chem. Phys. 8, 26 (2006).

${ }^{15}$ D. Holland and D. Shaw, Chem. Phys. 409, 11 (2012).

${ }^{16}$ H. Wollnik, J. Mass Spectrom. 34, 991-1006 (1999).

${ }^{17}$ J. J. Lin, J. Zhou, W. Shiu, and K. Liu, Rev. Sci. Instrum. 74, 2495 (2003).

${ }^{18}$ M. L. Lipciuc, J. B. Buijs, and M. H. M. Janssen, Phys. Chem. Chem. Phys. 8, 219 (2006).

${ }^{19}$ A. Vredenborg, W. G. Roeterdink, and M. H. M. Janssen, Rev. Sci. Instrum. 79, 063108 (2008).

${ }^{20}$ S. Manzhos and H.-P. Loock, Rev. Sci. Instrum. 75, 2435 (2004).

${ }^{21}$ W. A. Stein and the Sage Development Team, SAGE mathematics software, Ver. 5.3 (2012), see http://www.sagemath.org.

${ }^{22}$ See supplementary material at http://dx.doi.org/10.1063/1.4793404 for a lin-BASEX evaluation code.

${ }^{23}$ S. Eden, P. Limão-Vieira, P. Kendall, N. Mason, J. Delwiche, M.-J. HubinFranskin, T. Tanaka, M. Kitajima, H. Tanaka, H. Cho, and S. Hoffmann, Chem. Phys. 297, 257 (2004).

${ }^{24}$ J. Harvey, A. Bodi, R. P. Tuckett, and B. Sztáray, Phys. Chem. Chem. Phys. 14, 3935 (2012).

${ }^{25}$ J. Harvey, R. P. Tuckett, A. Bodi, and P. Hemberger, "Vibrational and electronic excitations in fluorinated ethene cations from the ground up," $\mathrm{J}$. Chem. Phys. (submitted).

${ }^{26}$ A. Bodi, P. Hemberger, T. Gerber, and B. Sztáray, Rev. Sci. Instrum. 83, 083105 (2012). 